\title{
Determining the Connection between Capacitively Coupled Electrocardiography Data and the Ground Truth
}

\author{
Anna Böhm, Christoph Hoog Antink, Steffen Leonhardt, Daniel Teichmann \\ Philips Chair for Medical Information Technology, RWTH Aachen, Germany
}

\begin{abstract}
Capacitively coupled electrocardiography (cECG) is a means of measuring the electrocardiogram (ECG) unobtrusively. Thus, it has a substantial advantage over the standard ECG using galvanic electrodes, which have to be placed directly on the skin of the patient potentially causing skin irritations and inconvenience. Nevertheless, cECG has multiple drawbacks concerning signal quality and is therefore not used in a clinical environment yet. One of the problems regarding cECG is the different morphology of the signal. As a consequence, clinical diagnoses from the cECG are challenging. In this paper, an approach is pursued to estimate the actual ECG from a given cECG. A method based on a multiple-input-single-output deconvolution technique is presented. The aim is to reconstruct a single-lead reference ECG from multiple cECG channels. In order to test this approach, data from previous studies were analyzed. It was found that reconstruction via deconvolution of the reference ECG from three given $c E C G$ channels was indeed possible.
\end{abstract}

\section{Introduction}

Capacitively coupled ECG has been investigated by many groups in the past years [1]. Its benefit is a contactless measurement and therefore, among others, improved comfort for patients compared to the standard ECG. However, due to its capacitive coupling, the cECG signal differs from the conductive ECG in terms of morphology. Additionally, the cECGs amplitude is attenuated and it is more artifact-prone [2]. Hence, one difficulty of cECG, if no ground truth is available, is to deduce the reference ECG. The possibility to determine transfer functions based on mathematical modeling of the electrode-skin contact and the knowledge of the capacitive ECG system has been shown in the past [3]. However, given such a model, it is difficult to correctly estimate its parameters.

The deconvolution algorithm is based on [4] and its application to vital signs was shown in [5]. The latter de- scribes a single input multiple output (SIMO) deconvolution method applicable for cardiac signals. The SIMO deconvolution process is performed in the frequency domain, where convolution becomes a simple multiplication.

The initial assumption that this deconvolution concept can be used for cardiac signals, is based on the knowledge that heart activity is the source of multiple bio-signals. These bio-signals may be recorded electrically, optically or mechanically. The resulting signals look very different but ultimately have the same cardiac source and are therefore delivering the same information. On this basis, Hoog Antink et al. assume cardiac activity as a train of impulses [5]. These impulses are delayed and filtered to result in different bio-signals: ECG, photoplethysmogram (PPG), ballistocardiogram (BCG), etc. Each modality can be calculated from the impulses by a determined delay, a "transfer" filter and Gaussian noise. It was shown that an estimation of these filters from the given multi-modal synthetic as well as recorded data is possible [5].

This paper is structured as follows: in the methods section, the database and data selection, as well as the deconvolution algorithm are described. The next section presents the results. Finally, the last section consists of discussions and conclusions.

\section{Methods}

In this paper, an approach based on a multiple-inputsingle-output (MISO) deconvolution technique is presented. The aim is to reconstruct a single-lead reference ECG from multiple cECG channels.

The algorithm was tested on data from previous studies.

\subsection{Database and Data Selection}

cECG data were selected from the MedIT UnoVis database [6]. The chosen recordings consisted of three cECG and one reference ECG channel from several test subjects that were acquired with a driver's seat equipped with six cECG electrodes during test drives. The experimental setup is described in detail in [6]. In total, the dataset UnoVis_auto2012 contains 13.4 h of data. 


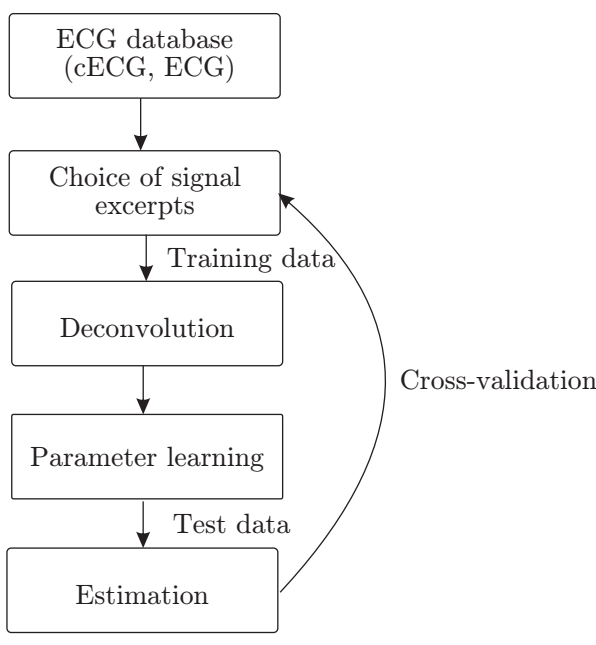

Figure 1. Signal processing realization

Since large parts of the data were contaminated with noise, preselection was conducted. For this purpose, a multichannel algorithm was used [7] [8]. Beat-to-beat intervals from multiple channels were be estimated. If it was not possible to estimate intervals, it was concluded that an artifact was present across all channels. These were therefore discarded. Additionally, in order to obtain larger coherent parts of the signal, at least 18 consecutive beats had to be detected to qualify for the data set.

Subsequently, test set and training set were selected for 10 -fold cross validation. Random coherent parts of the database were selected for ten distinct groups. Each of the groups was test set for one round, the rest was used as training data, see Figure 1.

\subsection{Deconvolution Algorithm}

The underlying model for the deconvolution is presented in Figure 2. The reference signal is estimated from three cECG signals that are delayed and filtered. In order to obtain the estimation, the filters $\vec{A}_{i}$ of order $q$ and with $i=\{1,2, \ldots, m\}$ channels are calculated. The delays $e^{-j \omega T}$ are estimated using cross-correlation.

If $y(t)$ is the reference ECG, $x_{i}(t)$ are the capacitive ECG channels where $n(t)$ represents noise and $t \in$ $\{0, \ldots, T\}$. The filter coefficients are denoted $a_{i}(\tau)$. Then

$$
y(t)=\sum_{i=1}^{m} \sum_{\tau=0}^{q} x_{i}(t-\tau) a_{i}(\tau)+n(t) .
$$

Now the Fourier transform $Y(\omega)$ can be calculated:

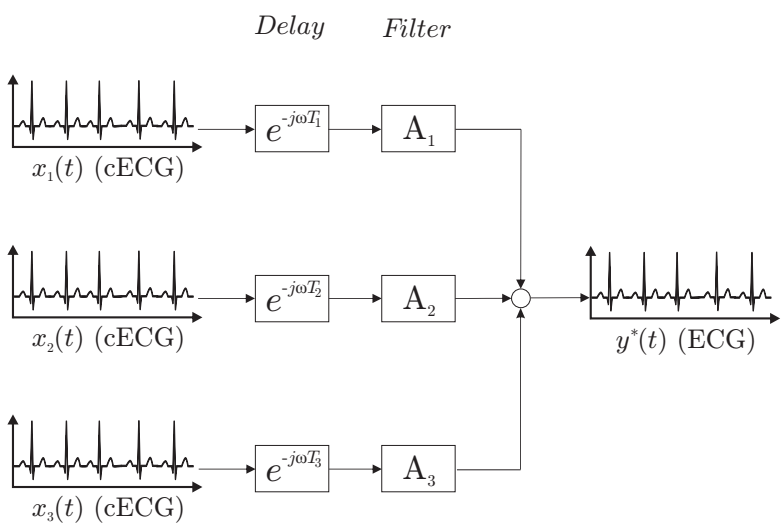

Figure 2. Estimation of the reference ECG signal from 3 cECG signals

$$
Y(\omega)=\sum_{i=1}^{m} \vec{E}(\omega) X_{i}(\omega) \cdot \vec{A}_{i}+N(\omega),
$$

with the filter vectors $\vec{A}_{i}$ for each cECG channel:

$$
\overrightarrow{A_{i}}=\left(a_{1}(1), \ldots, a_{1}(q)\right)^{T} .
$$

$\vec{E}(\omega)$ signifies the delay vector:

$$
\vec{E}(\omega)=\left(1, e^{-j 2 \pi \omega / T}, \ldots, e^{-j 2 \pi \omega q / T}\right) .
$$

Using $\vec{E}(\omega)$ the matrix $\mathbf{E X}$ is constructed,

$\mathbf{E X}=\left(\begin{array}{cccc}\vec{E}(0) \cdot X_{1}(0) & \vec{E}(0) \cdot X_{2}(0) & \ldots & \vec{E}(0) \cdot X_{m}(0) \\ \vec{E}(1) \cdot X_{1}(1) & \vec{E}(2) \cdot X_{2}(1) & \ldots & \vec{E}(1) \cdot X_{m}(1) \\ \ldots & \ldots & \ldots & \ldots \\ \vec{E}(T) \cdot X_{1}(T) & \vec{E}(T) \cdot X_{2}(T) & \ldots & \vec{E}(T) \cdot X_{m}(T)\end{array}\right)$

and the multi-channel filter vector

$$
\vec{A}=\left(\begin{array}{c}
\overrightarrow{A_{1}} \\
\overrightarrow{A_{2}} \\
\cdots \\
\overrightarrow{A_{m}}
\end{array}\right)
$$

The goal is now to find the minimal solution for

$$
\underset{\vec{A}}{\operatorname{argmin}}\|\mathbf{E X} \vec{A}-\vec{Y}\|_{2}^{2} .
$$

After separating real- and imaginary part, the leastsquares optimal solution is

$$
\vec{A}=\left(\mathbf{E X}^{T} \mathbf{E X}\right)^{-1} \mathbf{E X}^{T} \cdot \vec{Y} .
$$


The estimated Fourier transform of the reference ECG is

$$
\vec{Y}^{*}=\mathbf{E X} \cdot \vec{A}
$$

The time-domain estimated signal $y^{*}(t)$ is calculated by inverse transformation of $\vec{Y}^{*}$.

\section{Results}

We applied deconvolution to our test data and obtained an estimated ECG signal. By using cross-correlation it became clear that the delays between $\mathrm{cECG}$ and ECG were found to be negligible. In terms of delay, the cECG and ECG are congruent, which is reasonable since both signals share the same electrical source. There might be a slight difference, but it cannot be accurately recorded with a sample rate of $100 \mathrm{~Hz}$.

In Figure 3 an excerpt of the test data is shown with the filter length $q=75$ and a sample rate of $f=100 \mathrm{~Hz}$. The three cECG channels and the estimation are plotted with the reference ECG. It can be seen that the R-peaks of the cECG channels are lower than the reference. In channel 1, the R-peak is half the size of the reference. In contrast, the estimation's R-peak is higher. Additionally, the S-waves are too negative compared to the gold standard. However, this is not corrected in the estimated ECG. Furthermore, artifacts occur in this excerpt in the cECG channels. In channels 1 and 3, an artifact occurs during the second Qwave, which is not visible in the estimated ECG.

In order to evaluate the results, the estimation was performed with 10-fold cross-validation. The root-meansquare error deviation (RMSE) and the correlation are depicted in Table 1. The error was the highest in channel 1 followed by channel 2 of the capacitive ECG. Channel 3 was the best capacitive ECG, but it performed worse than the estimated ECG in every test run that was conducted.

\section{Discussion and Conclusion}

The RMSE and the correlation of the reference ECG signal to its estimation obtained via deconvolution were an improvement over each of the unprocessed cECG channels. While this is a promising result, its applicability to other cECG setups has to be shown in future studies. Overall, it was shown that the estimation of the reference ECG from multiple cECG channels is possible with deconvolution.

\section{Acknowledgments}

Funded by the Excellence Initiative of the German federal and state governments.

\section{References}

[1] Chi YM, Jung TP, Cauwenberghs G. Dry-contact and noncontact biopotential electrodes: Methodological review. IEEE Reviews in Biomedical Engineering 2010;3:106-119. ISSN 19373333.

[2] Wartzek T, Lammersen T, Eilebrecht B, Walter M, Leonhardt S. Triboelectricity in capacitive biopotential measurements. IEEE Transactions on Biomedical Engineering 2011; 58(5):1268-1277. ISSN 00189294.

[3] Eilebrecht B. Kapazitive EKG-Messtechnik für Diagnostik und für die Überwachung der Arbeitsbelastung. Shaker, 2014.

[4] He ZS, Xie SL, Fu YL. A new blind deconvolution algorithm for SIMO channel based on neural network. $2005 \mathrm{In}$ ternational Conference on Machine Learning and Cybernetics 2005;6.

[5] Hoog Antink C, Bruser C, Leonhardt S. Multimodal sensor fusion of cardiac signals via blind deconvolution: A sourcefilter approach. In Computing in Cardiology Conference (CinC). Boston, 2014; 805-808.

[6] Wartzek T, Czaplik M, Antink CH, Eilebrecht B, Walocha R, Leonhardt S. UnoViS: the MedIT public unobtrusive vital signs database. Health Information Science and Systems 2015;3(1):2. ISSN 2047-2501.

[7] Bruser C, Kortelainen JM, Winter S, Tenhunen M, Parkka J, Leonhardt S. Improvement of Force-Sensor-Based Heart Rate Estimation Using Multichannel Data Fusion. Biomedical and Health Informatics IEEE Journal of 2015;19:227235.

[8] Brüser C, Stadlthanner K, De Waele S, Leonhardt S. Adaptive beat-to-beat heart rate estimation in ballistocardiograms. IEEE Transactions on Information Technology in Biomedicine September 2011;15(5):778-786. ISSN 10897771.

Address for correspondence:

Anna Böhm

Pauwelsstr. 20

52074 Aachen

Germany

boehm@hia.rwth-aachen.de 

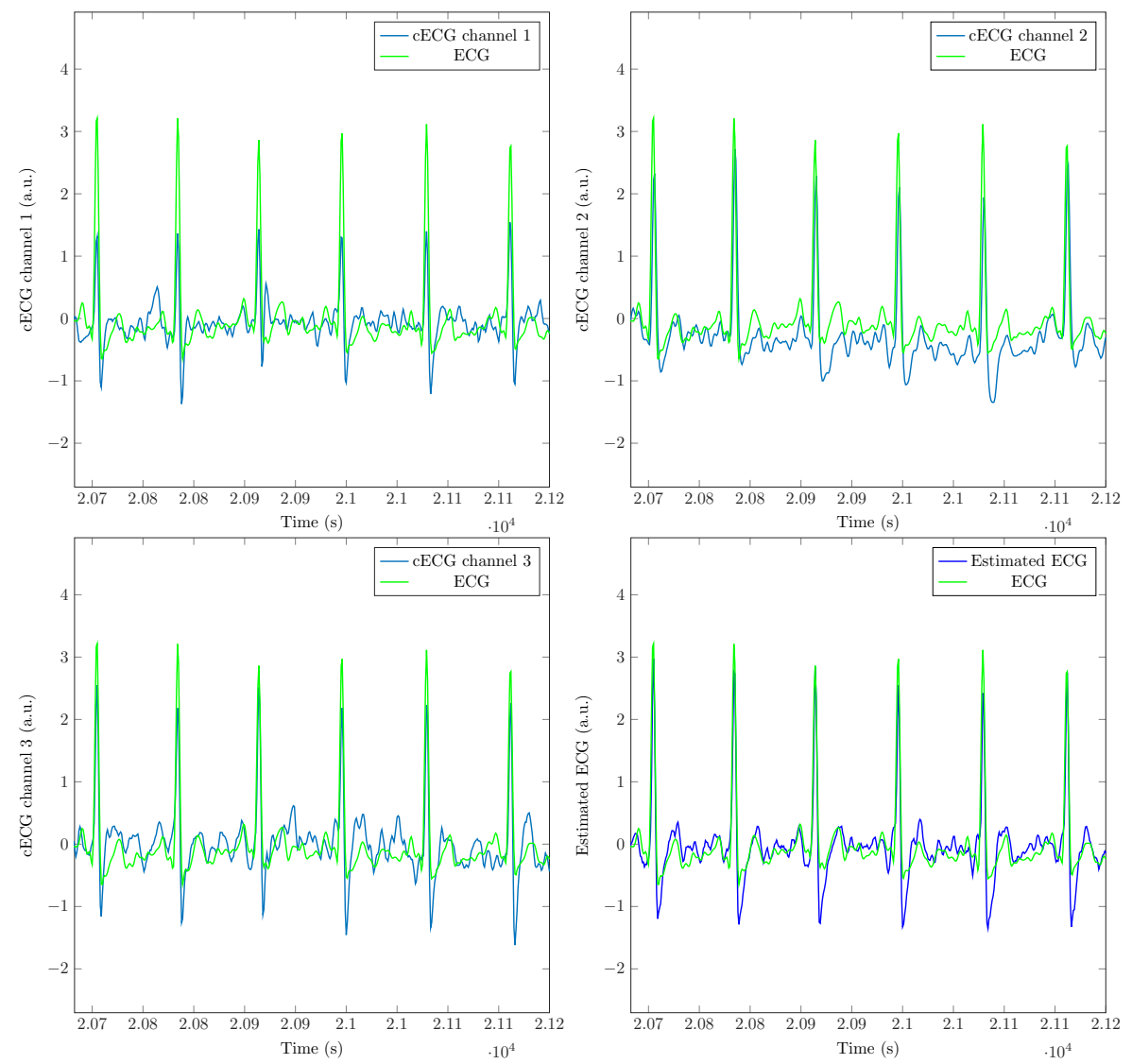

Figure 3. Capacitive ECG channels compared with the estimated ECG and reference ECG

Table 1. RMSE and correlation of the reference ECG with the capacitive ECG channels 1,2,3 and the estimation from deconvolution

\begin{tabular}{lllllllll}
\hline \multicolumn{3}{c}{ RMSE } & \multicolumn{7}{c}{ Correlation } \\
Data & cECG 1 & cECG 2 & cECG 3 & Estimation & cECG 1 & cECG 2 & cECG 3 & Estimation \\
\hline Run 1 & 1.2880 & 1.0193 & 0.7337 & 0.6119 & 0.1409 & 0.4611 & 0.7216 & 0.7742 \\
Run 2 & 1.1171 & 0.9600 & 0.7302 & 0.5667 & 0.3554 & 0.5243 & 0.7250 & 0.8107 \\
Run 3 & 1.2517 & 1.0606 & 1.0726 & 0.6954 & 0.1910 & 0.4192 & 0.4063 & 0.6982 \\
Run 4 & 1.1862 & 0.9241 & 0.7610 & 0.5677 & 0.2747 & 0.5599 & 0.7018 & 0.8119 \\
Run 5 & 1.2611 & 1.0333 & 0.7506 & 0.5959 & 0.1776 & 0.4477 & 0.7090 & 0.7874 \\
Run 6 & 1.0874 & 1.0441 & 0.8722 & 0.6898 & 0.3940 & 0.4412 & 0.6104 & 0.7110 \\
Run 7 & 1.3136 & 1.0734 & 0.8288 & 0.6491 & 0.1065 & 0.4032 & 0.6444 & 0.7401 \\
Run 8 & 1.2438 & 1.0931 & 0.7055 & 0.6499 & 0.2004 & 0.3824 & 0.7431 & 0.7420 \\
Run 9 & 1.2122 & 0.9226 & 0.8601 & 0.5683 & 0.2415 & 0.5606 & 0.6182 & 0.8097 \\
Run 10 & 1.2499 & 1.0313 & 0.8280 & 0.6221 & 0.1967 & 0.4530 & 0.6476 & 0.7686 \\
\hline Total & 1.2211 & 1.0162 & 0.8143 & 0.6217 & 0.2279 & 0.4653 & 0.6527 & 0.7654 \\
\hline
\end{tabular}

